\title{
SCSC metal-organic framework transmetallation: a successful case
}

\author{
$\underline{\text { Paolo Pio Mazzeo }}^{1}$, Stefano Canossa ${ }^{1}$, Paolo Pelagatti ${ }^{1}$, Alessia Bacchi ${ }^{1}$ \\ ${ }^{1}$ University Of Parma, Parma, Italy \\ E-mail: paolomazzeo.pm@gmail.com
}

Metal-Organic Frameworks (MOFs) are well known porous materials, extensively studied in the last decade and widely used in manifold applications such as gas absorption/storage, catalysis, controlled guest-delivery and many other [1].

A desired MOFs could be ex novo synthesized by direct reaction between metallic precursor and organic bridging ligand(s), usually via solvothermal reaction or, alternatively, it could be obtained via post-synthetic modification from an ad hoc starting framework [2].

Up to the present, the latter method is rarely described in the literature and only few successful examples of metallic node exchange have been reported.

A single-crystal-to-single-crystal Zn-to-Co metathesis is here proposed in a post-synthetic metal-organic framework modification performed in mild and reproducible experimental conditions.

The modified MOF shows a complete $\mathrm{Zn}$-to-Co transmetallation and results to be isostructural with respect to the starting framework, which only acted as a mould.

The new Co-MOF has been widely characterized via SR-SCXRD, SR-XRPD and ESEM analyses: results will be here described in details and compared with the native MOF.

In addition, the transmetallated as well as the native MOFs were then exposed to different environmental conditions: they both results to be temperature sensitive going through a similar reversible phase transformation which has been characterized via SCXRD.

A deep structure analysis will be also proposed with a description of the hypothesis of the active role of solvent/counter-ion in the transmetallation process.

An ex novo approach was also followed to obtain the Co-MOF via direct metal-ligand reaction but none of the synthetic routes proposed has given the same structure obtained via PSM of the native Zn-MOF; on the other hand, a non-porous more stable 1D coordination polymer was obtained.

A particular phase transformation of the 1D coordination polymer in which temperature and solvent play a synergistic role is also described.

[1] H. Furukawa et al. (2013). Science 341, 6149, 974

[2] M. Lalonde et al. (2013) J. Mater. Chem. A, 1, 5453

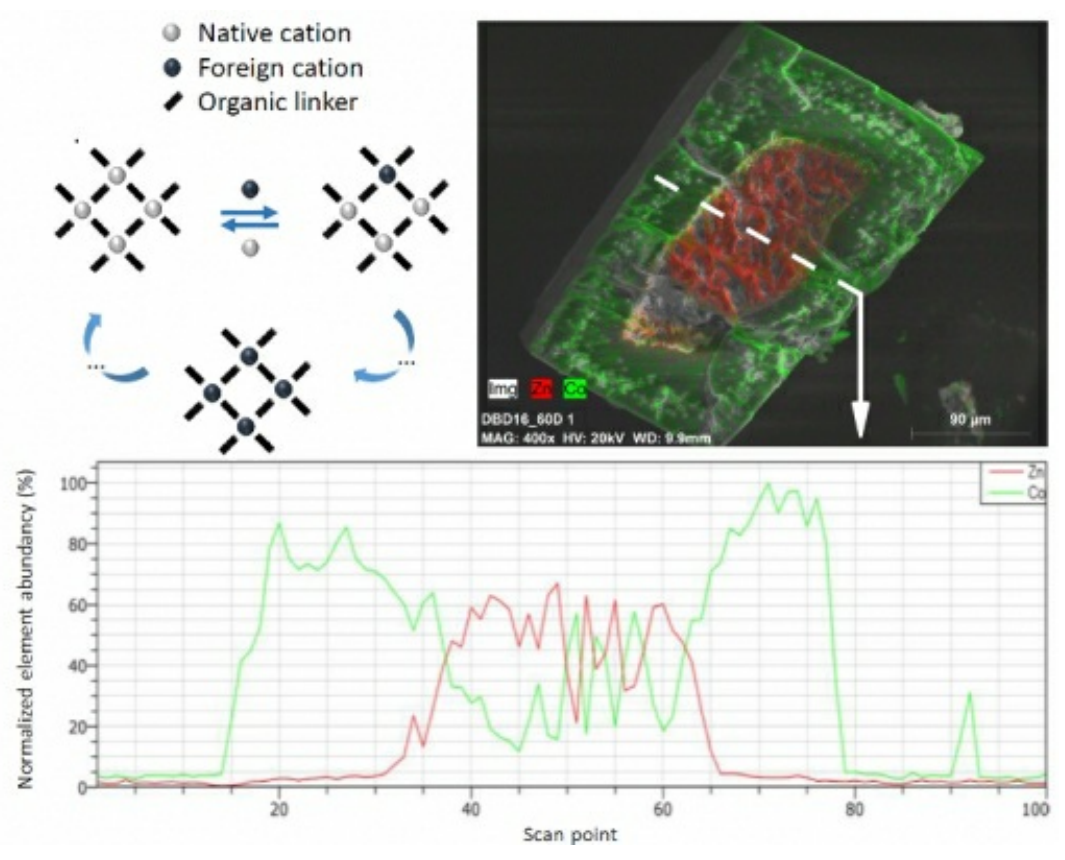

Keywords: Metal-Organic Frameworks, Transmetallation, Post-synthetic modification 